\title{
Transdermal Delivery of Insulin Using Trypsin as a Biochemical Enhancer
}

\author{
Ying-zhe Li, ${ }^{a}$ Ying-shu Quan, ${ }^{b, c}$ Lei Zang,${ }^{b}$ Mei-na $\mathrm{JIN}_{\mathrm{N}}{ }^{b}$ Fumio KamiYama, ${ }^{c}$ Hidemasa Katsumi,${ }^{b}$ \\ Akira Yамамото, ${ }^{b}$ and Sadami Tsutsumi ${ }^{*, a}$ \\ ${ }^{a}$ Institute for Frontier Medical Science, Kyoto University; Shogoin, Sakyo-ku, Kyoto 606-8507, Japan: ${ }^{b}$ Department of \\ Biopharmaceutics, Kyoto Pharmaceutical University; Misasagi, Yamashina-ku, Kyoto 607-8414, Japan: and ${ }^{c}$ CosMED \\ Pharmaceutical Co., Ltd.; 448-5 Kajii-cho, Kamigyo-ku, Kyoto 602-0841, Japan. \\ Received December 3, 2007; accepted May 19, 2008; published online June 4, 2008
}

Trypsin has been extensively used in laboratory settings for in vitro epidermal separation and keratinocyte isolation for over 50 years. The aim of this study was to assess the enhancing effect of trypsin on the transdermal delivery of insulin by applying its specific biochemical properties to react with the stratum corneum (SC) of skin. Bovine insulin was used as a model peptide to investigate in vitro permeation through rat skin and in vivo hypoglycemic effects of bovine insulin with or without the trypsin pretreatment. Trypsin significantly increased the transdermal permeability of bovine insulin in pH 3.0 solution, but no effect was observed in pH 6.0 solution. The permeation flux of bovine insulin from $\mathrm{pH} 3.0$ solution was promoted 5.2 -fold with $0.25 \%$ trypsin pretreatment when compared with the control. The enhancement of trypsin was dependent on the concentration in the range of $0.5-2.5 \%$. Furthermore, with trypsin pretreatment, the plasma glucose level was reduced to less than $60 \%$ of the initial value after $8 \mathrm{~h}$ of in vivo permeation of bovine insulin with $\mathrm{pH} 3.0$ solution, but did not return to the initial value during an 8-h experiment. Mechanistic studies with Fourier transform-infrared and attenuated total reflectance analysis and electrical resistance measurements suggest that trypsin alters the SC protein structure from the alpha- to the beta-form and decreases the electrical resistance of skin, thereby decreasing the SC barrier and enhancing the permeation of insulin. We conclude that trypsin would be effective as a biochemical enhancer for the transdermal delivery of peptide and protein drugs such as insulin.

Key words trypsin; transdermal; insulin; biochemical enhancer; plasma glucose level

There are 30 million patients with diabetes mellitus worldwide, and 1.2 million new patients are diagnosed each year, including a growing number of children and young adults. ${ }^{1)}$ However, insulin has been administered only by parenteral routes for the therapeutic control of type 1 diabetes; many patients typically require injections of insulin a minimum of two to three times per day. To improve patient convenience and compliance, alternative routes have been investigated including nasal, oral, transdermal, buccal, ocular, rectal, and vaginal pathways. ${ }^{2)}$

Transdermal delivery of insulin can prevent its metabolization and the first-pass effect and may thus be an optimal route of administration. However, delivery of large molecules by the transdermal route is also limited by the unique bioarchitecture of skin, which has primarily evolved as a protective barrier against the entry of microorganisms and water. ${ }^{3)}$ The stratum corneum (SC) barrier can be partially overcome by disruption of the lipid structure using fatty acids and surfactants as chemical penetration enhancers. ${ }^{4-6)}$ However, these enhancers are only useful for small molecules, ${ }^{6,7)}$ and it is difficult to achieve meaningful permeation of large peptides. Most recent research has focused on the use of electrical energy to increase drug flux across the skin, including techniques such as iontophoresis, sonophoresis, electroporation, etc. ${ }^{8-10)}$ Unfortunately, the large, complex, and relatively uncharged insulin molecule still cannot across the intercellular lipid layer of the SC. The transport rate of hexameric insulin by iontophoresis across mouse skin has been low and variable. ${ }^{11)}$ Monomeric insulin analogues carrying two additional negative charges have demonstrated transport rates 50 - 100-fold higher than those of hexameric insulin. However, the quantity of delivery is still not sufficiently high to provide an effective basal insulin level $(1 \mathrm{IU} / \mathrm{h}){ }^{11)}$

To overcome the barrier presented by the $\mathrm{SC}$, proteolytic enzymes maybe used as skin penetration enhancers because their action is highly specific toward proteins and their molecular size is too large to permeate the viable epidermis. Such enzymes perhaps alter skin permeability by provoking biochemical and metabolic events within skin. For more than 50 years, proteolytic enzymes such as trypsin have been extensively used in laboratory settings for in vitro epidermal separation and keratinocyte isolation. ${ }^{12-15)}$ The unique ability of proteases to cause selective epidermal separation has been in part explained by the proteolytic degradation of desmosomal proteins in the SC, which leads to cell dissociation. ${ }^{16,17)}$ Recently, several endogenous proteases occurring in the epidermis have been found to play important roles in regulating epidermal cell desquamation. ${ }^{18-21)}$ Based on these findings, several therapeutic applications have been attempted for wound debridement and epidermal ablation. ${ }^{2-24)}$ However, no studies have examined the use of such proteolytic enzymes as a penetration enhancers for the transdermal delivery of peptide and protein drugs such as insulin.

In the present study, a systematic investigation was undertaken to explore the possibility of the delivery of conventional bovine insulin using trypsin pretreatment in rats both in vitro and in vivo. Microscopy was employed to characterize visually the morphologic change in the skin. In addition, Fourier transform-infrared and attenuated total reflectance (ATR-FT-IR) analysis and electrical resistance measurement were performed to examine the change in SC structure with trypsin treatment. 


\section{MATERIALS AND METHODS}

Materials Trypsin and bovine insulin were purchased from Nacalai Tesque Inc. (Kyoto, Japan). All other chemicals and reagents were of analytical grade.

Animals Male Wistar rats with body weight of 220$270 \mathrm{~g}$ were used for in vitro and in vivo studies. All experiments were approved by the local review board of Kyoto University and were conducted in accordance with the national and international guidelines for laboratory animal care.

In Vitro Permeation Studies Wistar rats were anesthetized by intraperitoneal injection of sodium pentobarbital $(40 \mathrm{mg} / \mathrm{kg}$ body weight). The abdominal hair of the rats was removed using an animal hair clipper, and full-thickness skin was excised. The excess fat adhering to the dermis side was removed using cotton. Franz diffusion cells with an effective diffusion area of $0.785 \mathrm{~cm}^{2}$ were used for the permeation study. A piece of excised rat skin was mounted between the receptor and donor chambers with the SC facing the donor compartment. Trypsin $0.25 \%, 0.5 \%, 1.0 \%$, and $2.5 \%(\mathrm{w} / \mathrm{v})$ solutions were prepared with PBS without calcium and magnesium salts $(0.1 \mathrm{M}, \mathrm{pH} 7.4$, PBS). Trypsin solution $200 \mu \mathrm{l}$ was administered to the donor compartment. After 30-min pretreatment, it was removed and the donor compartment was washed with a mixture of ethanol and PBS $(30: 70, \mathrm{v} / \mathrm{v})$ three times to protect against the decomposition of insulin by residual trypsin. As a control, $200 \mu \mathrm{l}$ of PBS was added to the donor compartment instead of trypsin solution. Subsequently, the donor compartment was supplemented with $200 \mu \mathrm{l}$ of bovine insulin solution $(2.0 \mathrm{mg} / \mathrm{ml}, \mathrm{pH} 3.0$ or $\mathrm{pH}$ $6.0)$. The receptor solution contained urea $(2.0 \mathrm{mg} / \mathrm{ml})$ as a disaggregating agent and agent to prevent adsorption to glass surfaces, as well as sodium azide $(0.0025 \%, \mathrm{w} / \mathrm{v})$ to prevent microbial growth. The cells were maintained at $37^{\circ} \mathrm{C}$ and stirred with magnetic bars. Receptor solution $100 \mu$ was withdrawn periodically and replaced with an equal volume of fresh receptor solution. The samples collected were analyzed using a computer-controlled gradient HPLC system (Hitachi 7000 , Japan) as reported previously. ${ }^{25}$

In Vivo Studies Diabetes was induced in Wistar rats by intraperitoneal injection of streptozotocin $(50 \mathrm{mg} / \mathrm{kg}$ body weight). The plasma glucose level (PGL) was determined at 2 and 3 weeks to ensure that diabetes had been induced. Only rats with PGL $>250 \mathrm{mg} / \mathrm{dl}$ were selected as diabetes model animals. Before the experiment, animals were fasted for $14 \mathrm{~h}$ and water was supplied ad libitum. On the day of the experiments, the rats were temporally anaesthetized with pentobarbitone sodium $(30 \mathrm{mg} / \mathrm{kg}$ body weight) and fixed in the supine position. An animal hair clipper was used to remove the abdominal hair. Trypsin $0.5,1.0$, and $2.5 \%(\mathrm{w} / \mathrm{v})$ solution impregnated in a $4 \times 4-\mathrm{cm}$ gauze pad was applied for pretreatment. After $30 \mathrm{~min}$, the trypsin-impregnated gauze was removed and the skin was wiped with diluted isopropyl alcohol. A bovine insulin solution ( $\mathrm{pH}$ 3.0) impregnated in a $4 \times 4-\mathrm{cm}$ gauze pad was applied; bovine insulin concentrations of 62.5 and $125 \mathrm{U} / \mathrm{ml}$ were used. The application site was covered with HiPAS dressing (CosMED Pharmaceutical Co. Ltd., Japan) and was further fixed using gum tape. Blood collected from the tail vein at predetermined time intervals up to $8 \mathrm{~h}$ was analyzed for PGL. The rats were allowed water freely during the experiment.
Histologic Examination The rats were anaesthetized with pentobarbitone sodium $(30 \mathrm{mg} / \mathrm{kg}$ body weight $)$ and fixed in the supine position. An animal hair clipper was used to remove the abdominal hair. Trypsin $0.5,1.0$, and $2.5 \%$ $(\mathrm{w} / \mathrm{v})$ solution impregnated in a $4 \times 4-\mathrm{cm}$ gauze pad was applied for pretreatment. After $30 \mathrm{~min}$, the skin was washed with PBS and excised. The skin piece was fixed in $10 \%(\mathrm{w} / \mathrm{v})$ formalin. A section was cut from each sample and stained with hematoxylin and eosin (H\&E) for microscopic examination. The treated skin sections were observed under a light microscope to assess the changes in skin structure caused by trypsin treatment.

ATR-FTIR Spectroscopy Analysis Rat skin was cut into pieces of $1.5 \times 6.0 \mathrm{~cm}$ and soaked in PBS or $1.0 \%(\mathrm{w} / \mathrm{v})$ trypsin solution for $5 \mathrm{~h}$. After treatment, the skin piece was blotted with tissue paper, dried at room temperature for 15 min, and subjected to ATR-FTIR analysis (JIR-MICRO6000, Japan). ATR-FTIR spectra were recorded in the frequency range of 4000 to $900 \mathrm{~cm}^{-1}$, with $4-\mathrm{cm}^{-1}$ resolution. Each spectrum was an average of 50 scans.

Electrical Resistance Measurements Platinum electrodes were introduced into the donor and receptor compartments of the diffusion cell to measure skin electrical resistance. Physiologic saline solution containing $2.5 \%(\mathrm{w} / \mathrm{v})$ trypsin was applied to the donor compartment, and the skin electrical resistance was measured every $15 \mathrm{~min}$ before and during enzyme application. To apply the electric field and measure the resistance, we used an impedance bridge (Model 12K, Mita Musen Kenkyusho Ltd., Japan). An AC electrical field of $1 \mathrm{kHz}$ was applied across the electrodes for $10 \mathrm{~s}$, and the skin electrical resistance was read. The saline resistance was measured separately without skin with the same assembly and was found to be sufficiently small not to affect the measured skin resistance. The ratio of the skin electrical resistance after enzyme application $(R)$ and before application $\left(R_{0}\right)$ gives the skin electrical reduction ratio $\left(R / R_{0}\right)$.

Data Analysis The cumulative amount of insulin permeated per unit of skin surface area was plotted against time, and the slope of the linear portion of the plot was estimated as the steady-state flux $\left(J_{\mathrm{ss}}\right)$. The permeability coefficient $P$ was calculated as $P=J_{\mathrm{ss}} / C_{0}$, where $C_{0}$ is the donor concentration of bovine insulin. The enhancement ratio of the permeability flux $(E R)$ can be expressed as $E R=J_{\text {with trypsin }} / J_{\text {control }}$, where $J_{\text {with trypsin }}$ is the permeation flux of insulin through the rat skin with trypsin, and $J_{\text {control }}$ is the permeation flux of insulin through the rat skin without trypsin.

Statistical Analysis Results are expressed as mean \pm S.E., and statistical significance was assessed using Student's $t$-test or Dunnett's test for multiple comparisons with $p<0.05$ as the minimum level of significance.

\section{RESULTS}

In Vitro Transdermal Permeation of Bovine Insulin with or without Trypsin Pretreatment Figure 1a shows the permeation profile of bovine insulin from solutions of different $\mathrm{pH}$ with or without trypsin pretreatment. The permeation of bovine insulin itself through the rat skin in a donor solution of $\mathrm{pH} 3.0$ was very low, and almost no permeation from a solution of $\mathrm{pH} 6.0$ occurred. However, a significant increase in permeation was achieved with a donor solu- 

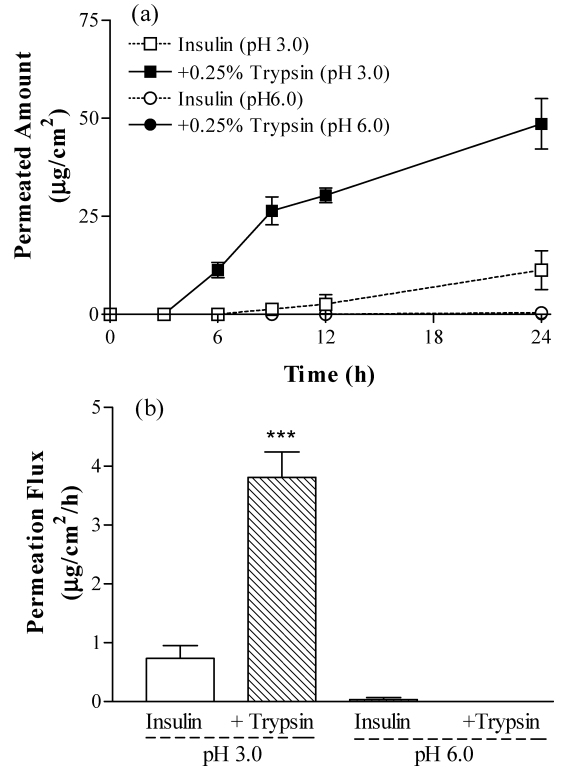

Fig. 1. In Vitro Transdermal Permeation of Bovine Insulin with Different $\mathrm{pH}$ Solutions through Rat Skin with or without $0.25 \%$ (w/v) Trypsin Pretreatment for $30 \mathrm{~min}$

(a) Permeation plots; (b) permeation flux. Each value represents the mean \pm S.E. of three experiments. $* * * p<0.005$ compared with the control (insulin alone).
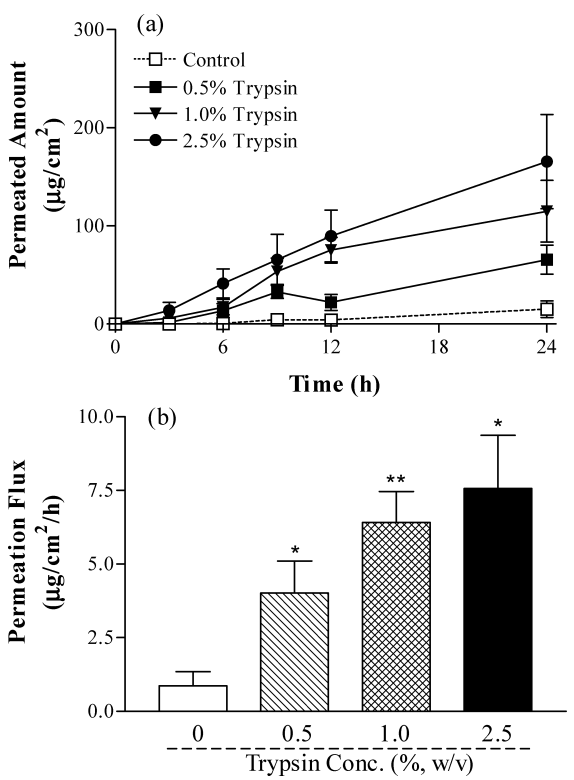

Fig. 2. Effects of Trypsin Concentration on the Transdermal Permeation of Bovine Insulin through Rat Skin with Pretreatment for $30 \mathrm{~min}$

(a) Permeation plots; (b) permeation flux. Each value represents the mean \pm S.E. of six experiments. $* p<0.05 ; * * p<0.01$ compared with the control (insulin alone).

tion of $\mathrm{pH} 3.0$ after pretreatment with $0.25 \%$ trypsin solution, although no enhancement was observed in the donor solution of $\mathrm{pH}$ 6.0. The permeability coefficient of bovine insulin from the donor solution of $\mathrm{pH} 3.0$ through the rat skin was increased from $1.02 \times 10^{-7}$ to $5.29 \times 10^{-7} \mathrm{~cm} / \mathrm{s}$, and the permeation flux was increased 5.2 -fold with $0.25 \%$ trypsin pretreatment (Fig. 1b). Figure 2 shows that the enhancement of trypsin was dependent on the pretreatment concentration $(0.5-2.5 \%)$. A higher concentration of trypsin shortened the lag time of insulin permeation. The permeation flux of insulin was $7.56 \mu \mathrm{g} / \mathrm{cm}^{2} / \mathrm{h}$ with $2.5 \%$ trypsin pretreatment, and

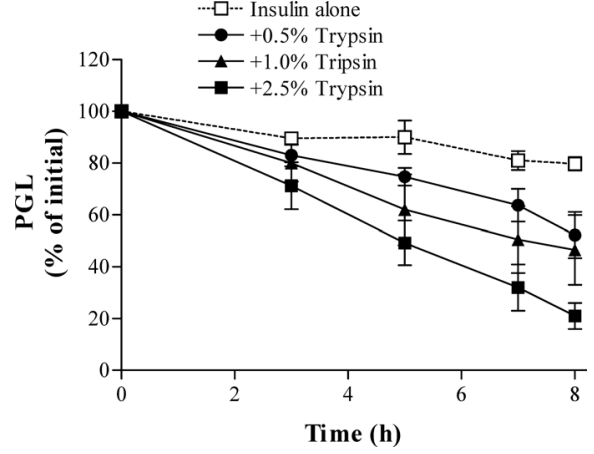

Fig. 3. Effects of Bovine Insulin $(62.5 \mathrm{U} / \mathrm{ml})$ on the PGL in Diabetic Rats with Different Concentrations of Trypsin Pretreatment for $30 \mathrm{~min}$ in Vivo

Each value represents the mean \pm S.E. of four experiments.

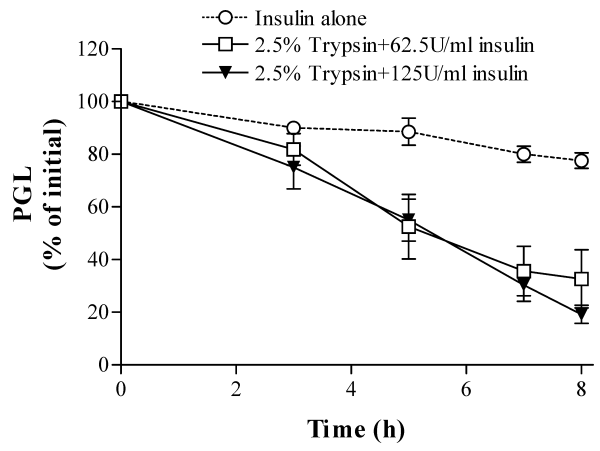

Fig. 4. Effects of Bovine Insulin Concentration on the PGL in Diabetic Rats with $1.0 \%(\mathrm{w} / \mathrm{v})$ Trypsin Pretreatment for $30 \mathrm{~min}$ in Vivo

Each value represents the mean \pm S.E. of five or six experiments.

the mean cumulative permeated amounts were 89.70 and $165.58 \mu \mathrm{g} / \mathrm{cm}^{2}$ at 12 and $24 \mathrm{~h}$, respectively.

In Vivo Hypoglycemic Effects of Bovine Insulin with or without Trypsin Pretreatment Based on the in vitro permeation results, bovine insulin solution of $\mathrm{pH} 3.0$ was used for in vivo studies. Figures 3 and 4 show the hypoglycemic effects of bovine insulin in diabetic rats with or without trypsin pretreatment. The application of bovine insulin alone without trypsin pretreatment did not cause a significant change in the PGL. However, a marked decrease in the PGL was observed in the group with trypsin pretreatment. The PGL was reduced to less than $60 \%$ of the initial value after $8 \mathrm{~h}$ in all groups with trypsin pretreatment, and these levels did not return to the baseline during the 8 -h experiment. The effects of trypsin on the hypoglycemic effects of bovine insulin were dependent on the pretreatment concentration (Fig. $3)$. In addition, we did not observe an insulin dose-dependent hypoglycemic effect with $1.0 \%$ trypsin pretreatment under the experimental conditions (Fig. 4).

Histologic Examination Photomicrographs of rat skin sections treated with PBS (control) or trypsin are shown in Fig. 5. In the control, a clearly defined SC could be seen (Fig. $5 \mathrm{a}$ ), but after treatment with trypsin, partial ablation and dilatation of the $\mathrm{SC}$ were observed (Figs. $5 \mathrm{~b}-\mathrm{d}$ ). Loosening of the SC was clearly induced by trypsin treatment.

ATR-FTIR Spectroscopy Because the SC is a complex matrix of protein and lipids, ATR-FTIR can be used for investigations of the protein composition and secondary structural changes in the SC after trypsin treatment. The two 
(a)

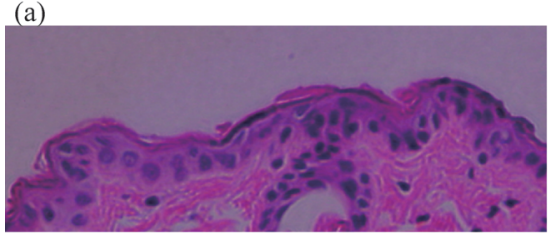

(c)

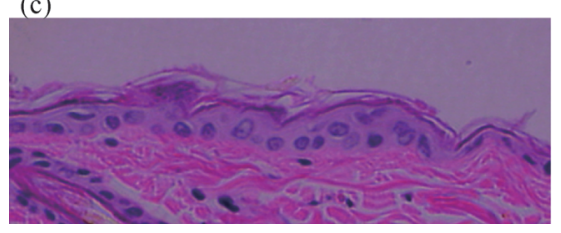

(b)

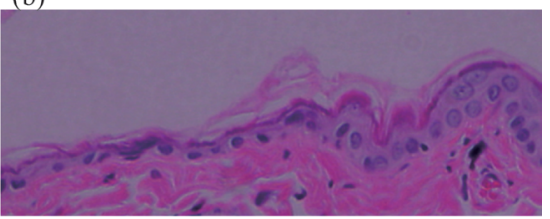

(d)

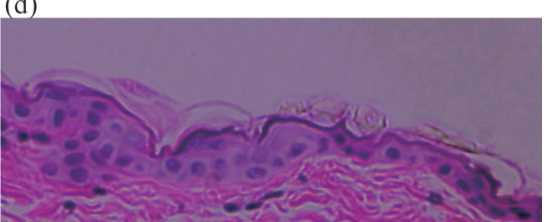

Fig. 5. Histologic Examination of Rat Skin with or without Trypsin Treatment in Vivo

(a) Rat skin treated with PBS for $30 \mathrm{~min}$; (b-d) rat skin treated with $0.5 \%, 1.0 \%$, or $2.5 \%(\mathrm{w} / \mathrm{v})$ trypsin solution for 30 min. Original magnification, $\times 200$.
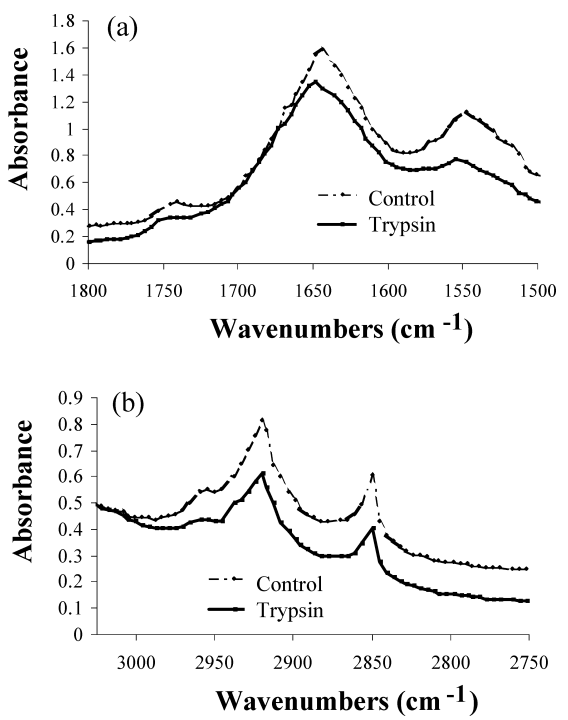

Fig. 6. ATR-FT-IR Spectrum of Rat Skin with PBS (Control) or $1.0 \%$ (w/v) Trypsin Treatment for $5 \mathrm{~h}$

strong absorption bands at around $1650 \mathrm{~cm}^{-1}$ (amide I) and $1550 \mathrm{~cm}^{-1}$ (amide II) are typical protein bands that arise mainly from $\mathrm{C}=\mathrm{O}$ stretching and $\mathrm{N}-\mathrm{H}$ bending vibrations, respectively, of the peptide. The peak at $1650 \mathrm{~cm}^{-1}$ is sensitive to protein conformation and varies as alpha-, beta-, or random conformations are introduced. ${ }^{26)}$ Asymmetric and symmetric $\mathrm{CH}_{2}$ vibration absorption bands at $2920 \mathrm{~cm}^{-1}$ and $2850 \mathrm{~cm}^{-1}$ are primarily due to the SC lipids. These bands are attributable to the long alkyl chains of ceramides and fatty acids, which are the major components of SC lipids. ${ }^{27,28)}$ The absorption at $1740 \mathrm{~cm}^{-1}$ is from esterified carbonyl groups corresponding to endogenous skin lipids. ${ }^{9)}$

Figures $6 \mathrm{a}$ and $\mathrm{b}$ show the ATR-FTIR spectrum of rat skin treated with PBS solution (control) or $1.0 \%$ trypsin solution for $5 \mathrm{~h}$. Compared with the control, some changes could be seen in the $1650 \mathrm{~cm}^{-1}$ region (amide I) and $1550 \mathrm{~cm}^{-1}$ region (amide II) in trypsin-treated skin samples (Fig. 6a). The amide I peak shift toward higher wave numbers (1643 to $1648 \mathrm{~cm}^{-1}$ ) and the amide II peak shift (1547 to $\left.1555 \mathrm{~cm}^{-1}\right)$ and a decrease in peak area were observed in trypsin-treated skin. These changes suggest disordering of the keratin structure. However, interestingly, there was almost no change in the SC lipid band in the $2920 \mathrm{~cm}^{-1}$ and $2850 \mathrm{~cm}^{-1}$ regions

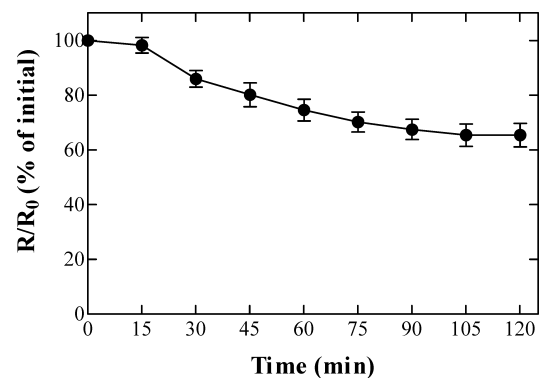

Fig. 7. Electrical Resistance of Rat Skin with or without $2.5 \%(w / v)$ Trypsin Treatment

when compared with the control (Fig. 6b). However, a slight change in the $1740 \mathrm{~cm}^{-1}$ region was observed.

Electrical Resistance Figure 7 shows that the electrical resistance of skin decreased after 15 min of trypsin treatment, and then slowly reached a plateau after $100 \mathrm{~min}$. The epidermal resistance of control was $1560.5 \Omega \cdot \mathrm{cm}^{2}$. After trypsin treatment, the epidermal resistance decreased to $65.4 \%$ of the initial value. The reduction in epidermal resistance suggests some changes in the secondary structures of the SC due to the trypsin treatment

\section{DISCUSSION}

Enzymes are essential components of all biological systems and they function as catalysts to accelerate the rate of specific biological reactions in living tissues. Within the epidermis, the endogenous SC proteases chymotrypic enzyme and tryptic enzyme normally present in the granular layer are known to hydrolyze intercorneocyte desmosomal adhesions $^{18,19)}$ and are believed to play an important role in epidermal desquamation. ${ }^{29)}$ It has been reported that the endogenous proteases trypsin and papain are capable of digesting intercellular desmosomal proteins in a similar fashion. ${ }^{30)} \mathrm{On}$ the molecular level, at least 75 potential tryptic cleavage sites have been predicted for the desmosomal proteins desmoglein 1 and $3 .^{31)}$ Trypsin can cleave peptides on the $C$-terminal side of lysine and arginine amino acid residues and it can also hydrolyze ester and amide linkages of synthetic derivatives of amino acids. Moreover, the in vitro enzymatic digestion of desmosomes might cause epidermal acantholysis and dissolution. ${ }^{16,17)}$ In the present study, we attempted to reduce the barrier properties of the SC by application of the above hy- 
drolytic mechanisms to promote the transdermal permeation of peptide and protein drugs.

In this study, we applied trypsin concentrations of $0.25 \%$ to $2.5 \%$ in in vitro and in vivo studies because these concentrations have been reported to be effective for the treatment of human epidermal ablation in vivo ${ }^{23}$ and are usually applied for epidermal separation and keratinocyte isolation. $^{12-15)}$ In in vitro permeation studies, a significant increase in the permeated amount of bovine insulin was obtained from $\mathrm{pH} 3.0$ solution after pretreatment with $0.25 \%$ trypsin, but no enhancement was observed with $\mathrm{pH} 6.0$ solution. It is known that insulin forms aggregates to varying degrees from dimers to hexamers depending on the $\mathrm{pH}$, concentration, and ionic strength of the solution. ${ }^{32)}$ These aggregates are larger in molecular size than monomeric insulin with a dimer of $12 \mathrm{kDa}$, a tetramer of $24 \mathrm{kDa}$, and a hexamer of $36 \mathrm{kDa}$. In the present study, the concentration of bovine insulin applied was $2 \mathrm{mg} / \mathrm{ml}$, with $\mathrm{pH}$ values of 3.0 and 6.0 . To clarify whether the different enhancing effects of trypsin with these two solutions of bovine insulin is associated with molecular size, we determined the molecular size and size distribution using dynamic light scattering (DLS) analysis. Interestingly, the mean hydrodynamic diameter of bovine insulin was $4.8 \mathrm{~nm}$, and the size distribution was in the range of $3.2-6.4 \mathrm{~nm}$ when the $\mathrm{pH}$ value was 3.0 at the above concentration. It has been reported that monomeric and hexameric insulin have diameters of 3.0 and $5.0 \mathrm{~nm},{ }^{11)}$ and hence bovine insulin in the solution with $\mathrm{pH} 3.0$ should exist mainly as a hexamer; there was an equilibrium among the different aggregated states as described by Jeffrey and Coates. ${ }^{33)}$ In addition, the bovine insulin solution of $\mathrm{pH} 6.0$ had a large size $(>5.0 \mathrm{~nm})$; bovine insulin was further aggregated and even precipitated, resulting in lower skin diffusivity and mobility. Based on these in vitro results, we selected a donor solution of $\mathrm{pH} 3.0$ for in vivo transdermal delivery of insulin with trypsin pretreatment.

In the in vivo studies, we investigated the effects of trypsin on the hypoglycemic effects of bovine insulin in diabetic rats and obtained a marked decrease in the PGL with bovine insulin solution of $\mathrm{pH} 3.0$ using trypsin pretreatment. Many studies have reported that iontophoresis of insulin can reduce the PGL in diabetic rats. Kanikkannan et al. reported that the onset of action with iontophoresis of a monomeric human insulin analogue was rapid $(2 \mathrm{~h})$, but that the PGL gradually reached the initial level during an 8 -h experiment. ${ }^{8)}$ Pillai and Panchagnula observed that terpene/ethanol caused synergistic enhancement of insulin permeation when combined with iontophoresis. ${ }^{34)}$ On the other hand, Kanikkannan et al. did not obtain any reduction in the PGL with oleic acid and methanol pretreatment followed by iontophoresis of insulin. ${ }^{8}$ In our study, the PGL reduction effect was continuous following application and did not return to the initial value during the 8-h experimental period. This result agrees with the finding of increased cumulative bovine insulin permeation during the in vitro permeation experiment. Insulin is extensively cleaved by various luminal and cellular enzymes such as pepsin, trypsin, $\alpha$-chymotrypsin, carboxypeptidases, and aminopeptidases. $^{35,36)}$ In this study, we used diluted isopropyl alcohol to wipe the trypsin-treated skin for removing and inhibiting the residual enzyme. In our previous study, $>30 \%$ alcohol protected insulin from cleavage by skin enzymes in the skin homogenizing solution (unpublished), but did not affect its solubility and activity. In all of our experiments with insulin including in vitro and in vivo studies, 30\% ethanol and the dilute isopropyl were also applied to the control group, and hence the effects of these alcohols on the skin and their influence on the enhancing effect of trypsin should be negligible.

In addition, we did not obtain a dose-dependent effect of insulin on the PGL in the present study. Many studies showed no significant effect of insulin concentration $(0.2$ $10 \mathrm{mg} / \mathrm{ml})$ on PGL reduction in diabetic rats. ${ }^{8,37)}$ In the present study, we confirmed that the size distribution (3.3$5.0 \mathrm{~nm}$ ) was similar for $62.5 \mathrm{U} / \mathrm{ml}$ and $125 \mathrm{U} / \mathrm{ml}$ of insulin solutions, which could correspond to the permeability of insulin through the skin. On the other hand, we observed a concentration-dependent effect of trypsin on PGL reduction by insulin. This finding correlates with the in vitro concentration-dependent enhancing effects of trypsin on the transdermal permeation of insulin. With $0.5 \%$ and $1.0 \%$ trypsin pretreatment for $30 \mathrm{~min}$, the PGL was reduced to $40-50 \%$ of the initial value, but with $2.5 \%$ trypsin solution pretreatment for $30 \mathrm{~min}$, the PGL was reduced to $21 \%$ of the initial value. After $8 \mathrm{~h}$, we did not observe any skin irritation on the application sites except for minor swelling due to long exposure to the water-based solution. Continuous monitoring of these rats after the experiment showed no disorders in the application sites over the next $3 \mathrm{~d}$. Trypsin itself is a macromolecule consisting of a single-chain polypeptide of 223 amino acid residues including the active site with His and Ser. ${ }^{38,39)}$ Such a large enzyme cannot penetrate the barrier posed by an intact SC. ${ }^{23)}$

On ATR-FTIR analysis, the amide I and amide II peak shifts toward higher wave numbers in the $1650 \mathrm{~cm}^{-1}$ and $1550 \mathrm{~cm}^{-1}$ regions can be attributed to the rupture of peptide and disulphide bonds. ${ }^{40)}$ These changes indicate a decrease in the alpha-helix conformation accompanied by an increase in the disordered structures of keratin-filled SC corneocytes. Many approaches utilizing chemical enhancers and sonophoresis have been reported to enhance SC permeability, and mechanisms of skin permeation enhancement have also been examined. ${ }^{41-43)}$ The disordering of lipid bilayers is considered to be a major cause of the enhancement of skin permeability of drugs based on peak shifts toward higher wave numbers and/or increases in the band width of methylene stretching vibrations. ${ }^{43}$ However, the trypsin-treated skin showed changes neither in peak shift nor peak width when compared with the control skin in the methylene vibration region in the present study. These observations indicate that SC proteins, not lipid bilayers, may play an important role in insulin permeation with trypsin treatment. In addition, the $\mathrm{C}=\mathrm{O}$ stretching vibration at $1740 \mathrm{~cm}^{-1}$ results from ester linkage carbonyls within the lipid polar heads. The reason for the slight change in this region after trypsin treatment is unknown, but may be related to the structural reorganization of alkyl chains accompanied by restructuring of the bilayer near an ester linkage. However, this change was much smaller than that observed for amide I and amide II. Trypsin can cleave the bonds of keratin-filled corneocytes in the superficial area, thus loosening and disordering the secondary structures of cellular proteins especially at the surface of the corneocytes. In this regard, we observed under microscopy that 
the SC was swollen tremendously after trypsin treatment. This kind of loosening will be accompanied by the swelling of corneocytes and will form broader passways in the SC.

Electrical resistance of the skin is an average measure of the difficulty for charged molecules to traverse the skin. Changes in electrical properties also indicate some changes in the membrane properties of skin and thus they have been used to verify whether the structural integrity of skin samples is within the normal range. The monitoring of skin electrical resistance during trypsin treatment can provide insight into the mechanisms governing the enhancement of transdermal transfer. The decreased electrical resistance of the skin after trypsin treatment was considered to contribute to the enhancement of insulin delivery in our study. In most previous studies, applying low-frequency ultrasound was reported to decrease the electrical resistance of skin by a cavitation effect, which presumably disordered lipid bilayers and opened pathways. ${ }^{44)}$ Mitragotri et al. also found that cavitation inside the SC could potentially take place in keratinocytes, in the lipid regions, or both. ${ }^{45}$ Both of our results from ATR-FTIR analysis and electrical resistance measurement further verify that trypsin can disorder keratin-filled corneocytes and cause the reorganization of the peripheral lipids, thereby decreasing the barrier capability of skin and enhancing the transdermal delivery of insulin.

In this study, we applied trypsin as a biochemical enhancer for the transdermal delivery of insulin. With trypsin pretreatment, we achieved significant enhancement of transdermal permeation of bovine insulin in vitro and marked hypoglycemic effects of bovine insulin in vivo. Our results suggest that the permeation of bovine insulin with $2.5 \%$ trypsin pretreatment may provide $3.0 \mathrm{U} / \mathrm{h}$ with a $16 \mathrm{~cm}^{2}$ transdermal system, which is sufficient to treat diabetic rats. If the transdermal permeation area can be increased and further coapplication with other physical approaches can be achieved, this technique may be applied to human skin studies. We conclude that enhancement with trypsin pretreatment could be effective for transdermal delivery of peptide and protein drugs such as insulin.

\section{REFERENCES}

1) Sadrzadeh N., Glembourtt M. J., Stevenson C. L., J. Pharm. Sci., 96, 1925-1954 (2007).

2) Owens D. R., Zinman B., Bollit G., Diabetic Med., 20, 886-898 (2003).

3) Guy R. H., Pharm. Res., 13, 1765-1769 (1996).

4) Ashton P., Hadgraft J., Walters K. A., Pharm. Acta Helv., 61, 228 235 (1986).

5) Nishihata T., Rytting J. H., Takahashi K., Sakai K., Pharm. Res., 5, 738 -740 (1988)

6) Shokri J., Nokhodchi A., Dashbolaghi A., Hassan-Zadeh D., Ghafourian T., Barzegar J. M., Int. J. Pharm., 228, 99-107 (2001).

7) Issachar N., Gall Y., Borrel M. T., Poelman M. C., Contact Dermatitis, 39, 182-186 (1998).

8) Kanikkannan N., Singh J., Ramarao P., J. Controlled Release, 59, 99-
105 (1999)

9) Pillai O., Panchagnula R., Skin Pharmacol. Physiol., 17, 289-297 (2004).

10) Tokumoto S., Higo N., Sugibayashi K., Int. J. Pharm., 326, 13-19 (2006).

11) Langkjaer L., Brange J., Grodsky G. M., Guy R. H., J. Controlled Release, 51, 47-56 (1998).

12) Hentzer B., Kobayasi T., Acta Derm. Venereol., 58, 197-202 (1987).

13) Germain L., Rouabhia M., Guiganard R., Burns, 19, 99-104 (1993)

14) Kitano Y., Okada N., Br. J. Dermatol., 108, 555-560 (1983).

15) Liu S., Kaeasek M., J. Invest. Dermatol., 71, 157-162 (1978).

16) Egelrud T., Hofer P. A., Lundstrom A., Acta Derm. Venereol., 68, 93 97 (1988)

17) Komura J., Acta Derm. Venereol. Suppl., 73, 111-119 (1973).

18) Watkinson A., Harding C., Moore A., Coan P., Arch. Dermatol. Res. 293, 470-476 (2001).

19) Egelrud T., Acta Derm. Venereol., 208 (Suppl.), $44-45$ (2000).

20) Hansson L., Stromqvist M., Backman A., J. Biol. Chem., 269, 19420-19426 (1994).

21) Ekholm I. E., Brattsand M., Egelrud T., J. Invest. Dermatol., 114, 5663 (2000).

22) Falabella A. F., Carsonm P., Eagltein W. H., Flanga V., J. Am. Acad Dermatol., 39, 737-740 (1998).

23) Fein H., Maytin E. V., Mutasin D. F., Bailin P. L., Dermatol. Surg., 31, $139-148$ (2005).

24) Salcido R., Adv. Skin Wound Care, 13, 92 (2000).

25) Asada H., Douen T., Mizokoshi Y., Fujita T., Murakami M., Yamamoto A., Muranishi M., Pharm. Res., 11, 1115-1120 (1994).

26) Sznitowska M., Janicki A., Williams A., Lau S., Stolyhwo A., J. Pharm. Sci., 92, 173-179 (2003).

27) Cotte M., Dumas P., Besnard M., Tchoreloff P., Walter P., J. Controlled Release, 97, 268-281 (2004).

28) Vaddi H. K., Wang L. Z., Ho P. C. L., Chan Y. W., Chan S. Y., Chem Pharm. Bull., 49, 1395-1400 (2001).

29) Sondell B., Thornell L. E., Egelrud T., J. Invest. Dermatol., 104, 819 823 (1995).

30) Prunieras M., Arch. Dermatol. Res., 264, 243 -247 (1979).

31) Nilles L. A., Parry D. A., Powers E. E., J. Cell Sci., 99, 809-821 (1991).

32) Brange J., Langkjaer L., "Insulin Structure and Stability," ed. by Wang Y. J., Pearlman R., Plenum Press, New York, 1993, pp. 315-350.

33) Jeffrey P. D., Coates J. H., Biochemistry, 5, 3820-3823 (1966).

34) Pillai O., Panchagnula R., J. Controlled Release, 88, 287-296 (2003).

35) Smith E. L., Hill R. L., Borman A., Biochim. Biophys. Acta, 29, $207-$ 208 (1958).

36) Wolff A. M., Showell M. S., Venegas M. G., Adv. Exp. Med. Biol., 379, 113-120 (1996).

37) Pillai O., Borkute S. D., Sivaprasad N., Panchagnula R., Int. J. Pharm., 254, 271-280 (2003).

38) Walsh K. A., Methods Enzymol., 19, 41-63 (1970).

39) Keil B., "The Enzymes," 3rd ed., Vol. III, ed. by Boyer P. D., Academic Press, New York, 1971, pp. 250-275.

40) Carr C. M., Lewis D. M., Journal of Society of Dyers and Colourists, 109, 21-24 (1993).

41) Wojciechowska E., Rom M., Wlochowicz A., Wysocki M., Birczynska A. W., J. Mol. Struct., 704, 315-321 (2004).

42) Tanojo H., Junginger H. E., Bodde H. E., J. Controlled Release, 47, $31-39$ (1997).

43) Mak V. H. W., Potts R. O., Guy R. H., Pharm. Res., 7, 835-841 (1990).

44) Limary M. C., John M. T., Abedellaziz B. J., J. Pharm. Pharmacol., 56, 1109-1118 (2004).

45) Mirtagotri S., Edwards D. A., Blankschtein D., Langer R., J. Pharm. Sci., 84, 697-706 (1995). 